\title{
Standard visualization of biliopancreatic system by EUS
}

\section{Kida}

Kitasato University East Hospital

\section{Radial scanning}

\section{Introduction}

There are two visualization manner ( so called 'push method' and 'pull method') of biliopancreatic system, that is one is start from duodenal bulb, another from inferior duodenal angle. Although in case of pancreatic diseases we examine from the stomach body whether the pancreatic duct is dilated or not, generally we start EUS from from duodenal bulb. We suck air from the stomach and duodenum before filling a balloon with deaerated water and flow about $100 \mathrm{ml}$ of water into the duodenum before performing EUS examination. Liver and gall bladder are visualized in the upper half, the aorta and inferior vena cava in the right hand side (Fig. 1). After this we check all of the gall bladder by pulling (generally up to $55 \mathrm{~cm}$ from incisior) or pushing scope. However in case of a free gall bladder, it is visualized from the stomach body. After observation of the gall bladder we push the scope around the superior duodenal angle, and the portal vein

Correspondence: Mitsuhiro Kida, MD PhD · Dept. of Gastroenterology · Kitasato University · East Hospital · 2-1-1 Asamizodai · Sagamihara · Kanagawa · 2288520 Japan · Fax: +81-42-749-8690 · E-mail: m-kida@kirasato-uac.jp KG Stuttgart · New York · ISSN 0013-726X · DOI 10.1055/s-2006-946672

and bile duct are visualized in the left hand side, liver hilum in the upper half, and pancreas head-body and $2^{\text {nd }}$ portion of the duodenum in the lower half (Fig. 2). There are four possibility of ductal structure between EUS scope and portal vein, which include bile duct, cystic duct, proper hepatic artery, and main pancreatic duct. We can differentiate these ductal structure as follows: the bile duct and main pancreatic duct go into the pancreas, the proper hepatic artery out of the pancreas, and the cystic duct connects to the gall bladder. Then we follow the bile duct up to the duodenal papilla by controlling up and down or left and right angle (Fig. 3). Generally we can visualize the duodenal pappila with a longitudinal image of the bile duct and the main pancreatic duct in more than $50 \%$ of cases. However, if you feel resistance when you can not change the EUS picture by pushing the scope, you should stop pushing the scope. Otherwise you will make a perforation of the duodenum.

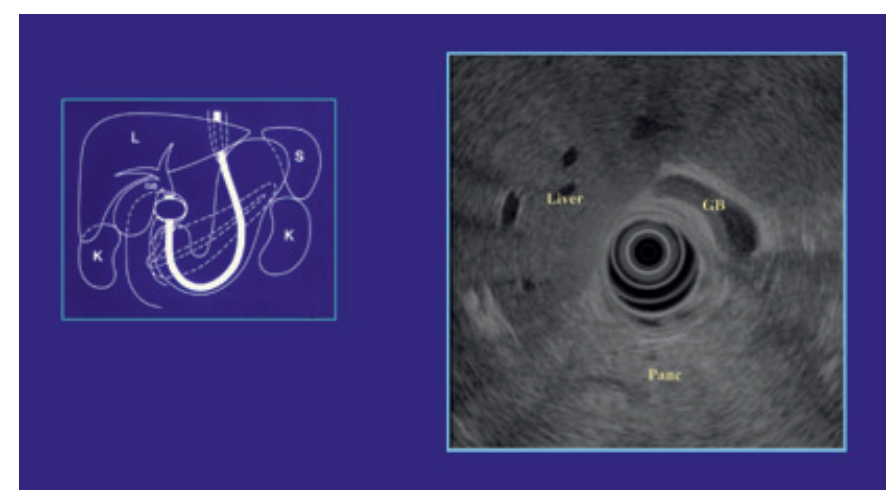

Fig. 1 From the duodenal bulb.

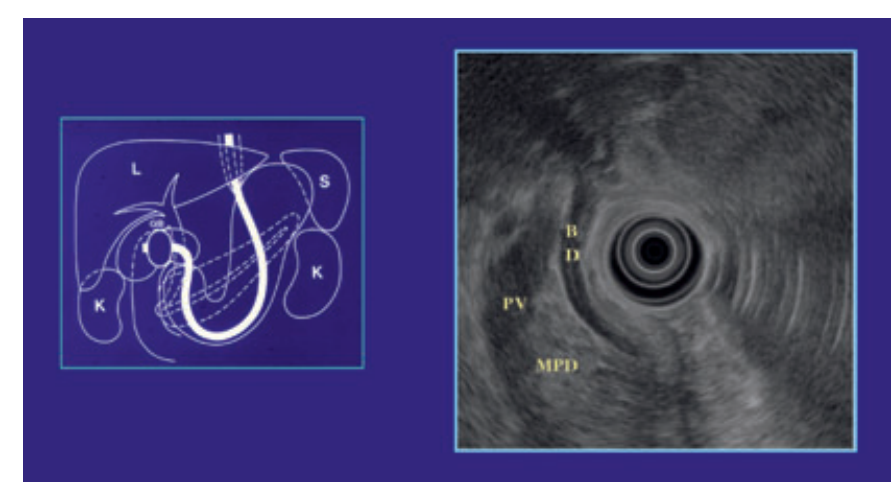

Fig. 2 From the superior duodenal angle.

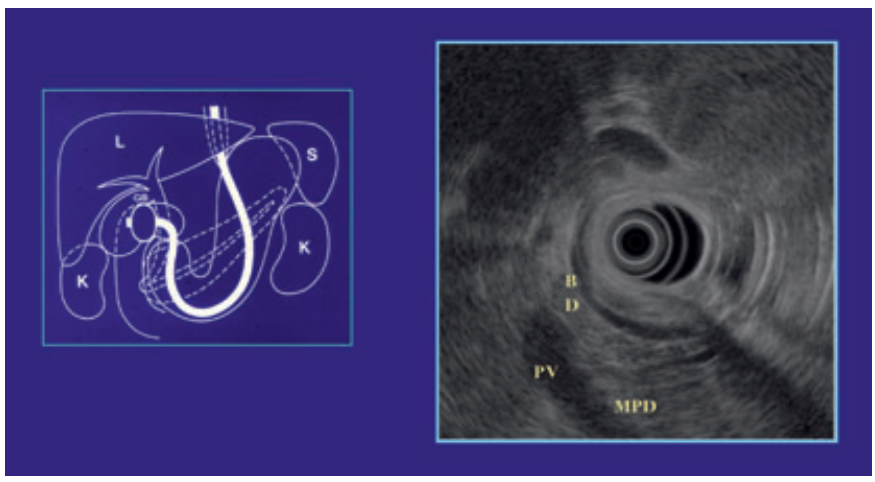

Fig. 3 From the superior duodenal angle. 


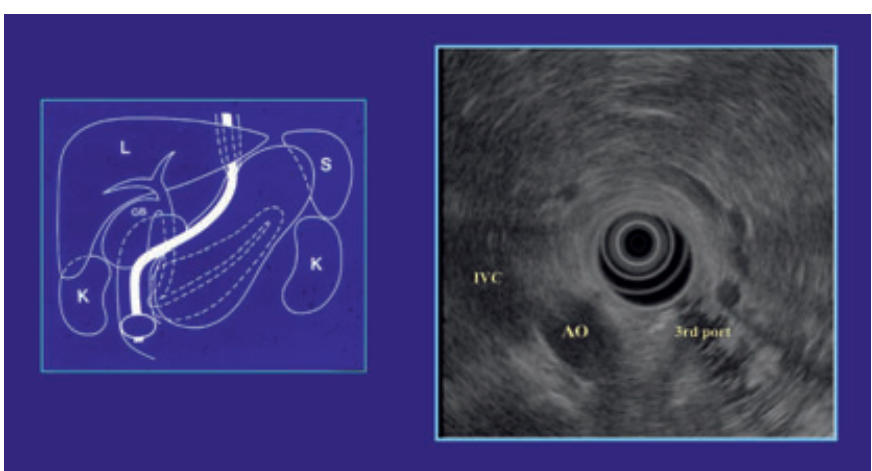

Fig. 4 From the inferior duodenal angle.

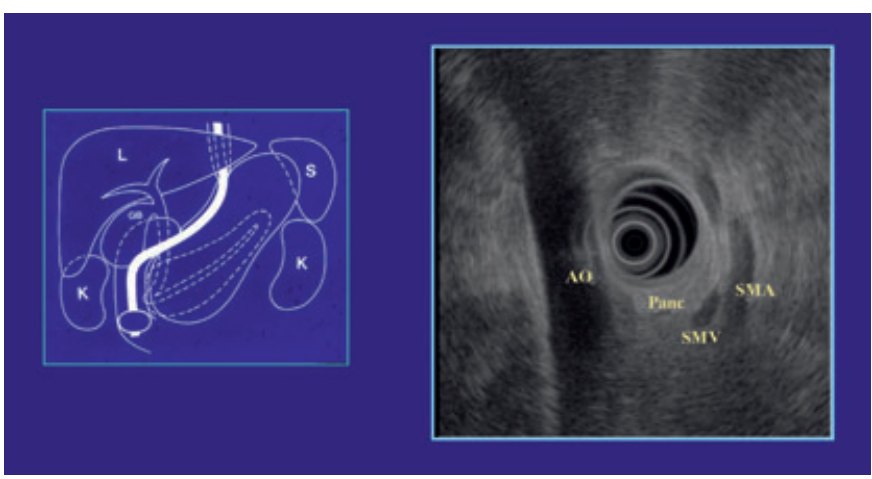

Fig. 5 From the inferior duodenal angle.

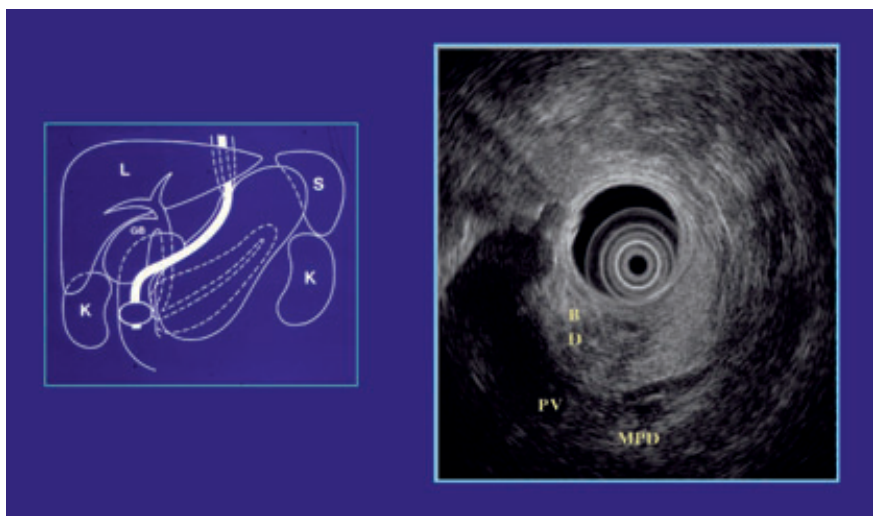

Fig. 6 From the $2^{\text {nd }}$ portion of duodenum.

From the inferior duodenal angle

Using stretch technique the EUS scope reaches the inferior duodenal angle with endoscopic guidance like that in endoscopic retrograde cholangiopancreatography. You should the suck air again and fill deaereated water into the duodenum, if necessary. You can find the aorta and vena cava in the direction of 6-9 o'clock. $3^{\text {rd }}$ portion of the duodenum in the direction of 5-6 o'clock, and you should control up-down or left-right angle to get transverse roud image of the aorta and inferior vena cava (Fig. 4). This means that the axis of body and scope is of the same. Then you manipulate the up angle to get longitudinal image of the aorta and or inferior vena cava, superior mescenteric vein in the left half and artery in the direction of 3-5 o'clock, and around the uncinate process of the pancreas is revealed in between, because the pancreas is located in the caudal side of $3^{\text {rd }}$ portion (Fig. 5). After observation of the uncinate process you pull the scope gradually and follow the pancreas, and then will find thickening of the duodenal wall (papilla) and low echoic pancreas next to the aorta (Fig. 6). Generally the pancreas near the papilla is revealed low echoic area, however nobody knows its reason. Then turn the left angle and up angle step by step and pull or push the scope gradually, not to miss the low echoic pancreas. After this manner you will get the longitudinal image of the bile duct and main pancreatic duct (this is pull method) like in Fig. 2 (Fig. 7), and you follow the bile duct up to the liver hilum and pull the scope into the duodenal bulb, and transitional part (genu) of the pancreas head and body is revealed next to the duodenum (Fig. 8). Generally, lesions in this area are easy to miss with EUS, therefore you should examine this area very carefully.

\section{From the stomach body}

Sucking water from the balloon of scope, the scope slip out to the stomach. Then you will fill deaereated water into the balloon

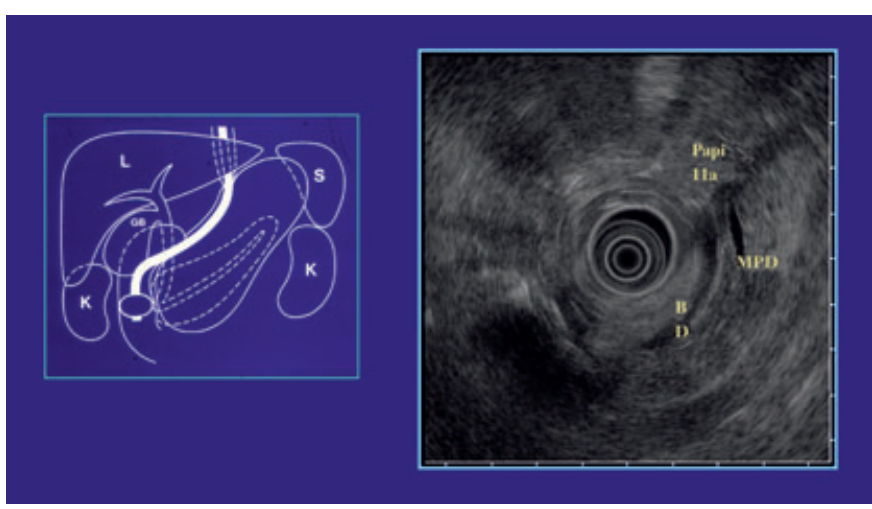

Fig. 7 From the $2^{\text {nd }}$ portion of duodenum.

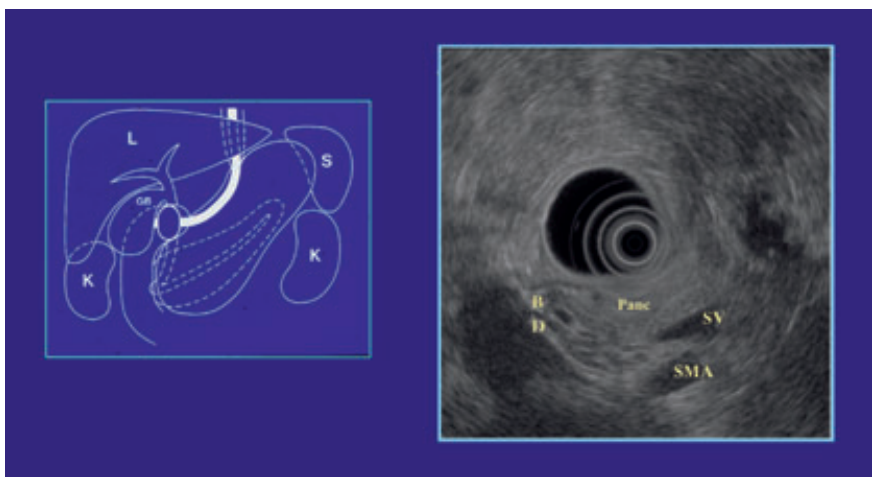

Fig. 8 From the duodenal bulb.

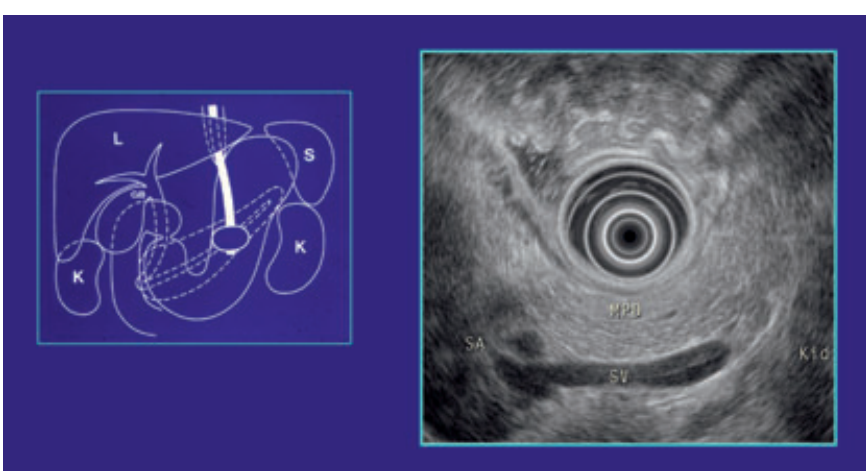

Fig. 9 From the stomach body. 


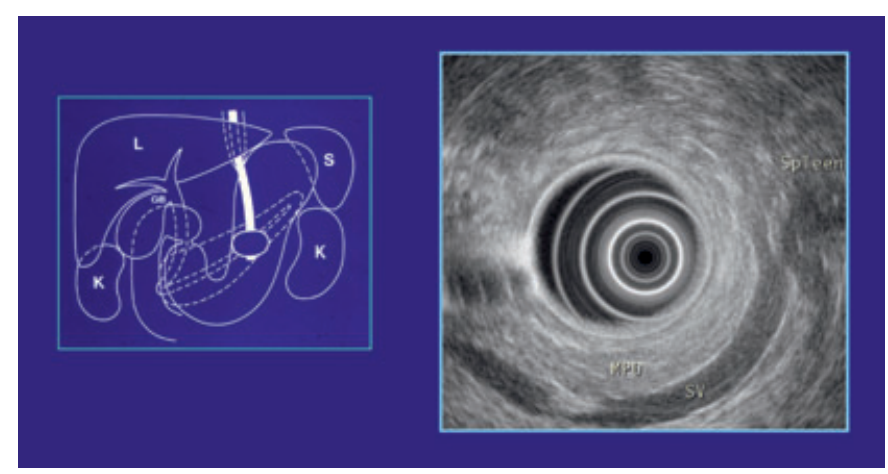

Fig. 10 From the stomach body.

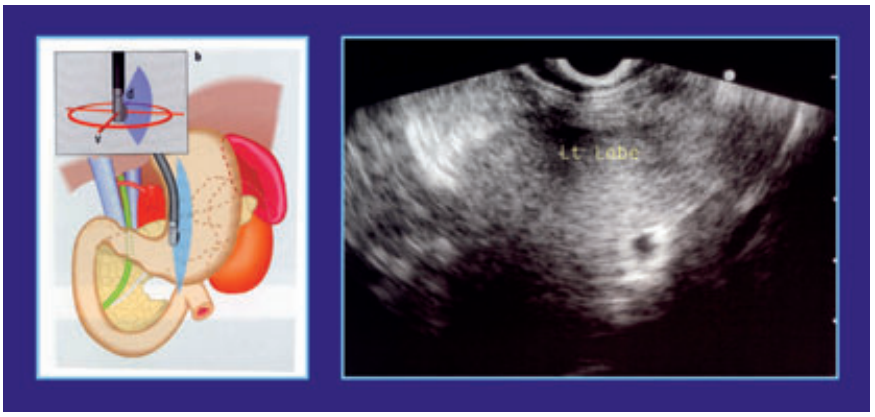

Fig. 11 From EG-Junction.

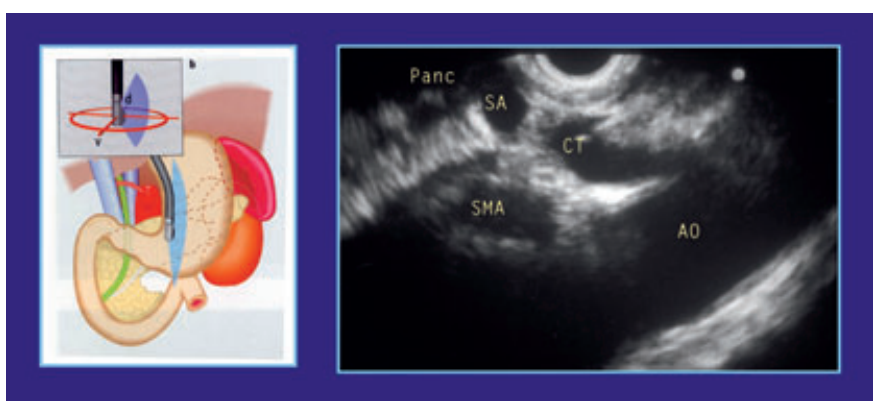

Fig. 12 From the stomach.

again and start scanning at the position of around $55 \mathrm{~cm}$ from the incisior. And you will find the portal and splenic vein, and the pancreas body is revealed in between these vein and the stomach wall (Fig. 9). Follow the pancreas up to the splenic hilum by turning to the right or right angle (Fig. 10). If you can not find the pancreas, you should start at the esophagocardiac junction, find the aorta, and you should follow the aorta by pushing the scope. Then you will find the celiac trunk, splenic, hepatic artery, and the pancreas, which is revealed in the slightly caudal side.

After observation of the pancreas body and tail, lymph node around celiac trunk should be checked. Using this standard visualization manner it takes from 15 to 30 minites to examine all of the biliopancreatic system with EUS.

\section{Convex scanning}

From the esophagocardiac junction

When we insert the scope up to the esophagocardic junction, the left lobe is generally revealed (Fig.11). We turn the scope up to
180 degree, then the aorta will be revealed, and follow it up to the celiac trunk. The pancreas body is revealed in between celiac trunk and the scope (Fig.12, Fig. 13). And we should follow the pancreas up to the splenic hilum and left kidney (Fig. 14). The left adrenal gland is revealed in between the pancreas tail and the kidney (Fig. 15). Then we push the scope up to the angle of stomach, and follow portal vein and bile duct up to the liver hilum.

From the duodenal bullb

When we push the scope in to the duodenal bulb, we can find the transitional part of the pancreas head and body, portal vein, and bile duct will be revealed by rotating the scope (Fig. 16, Fig. 17). And we follow the bile duct up to the papilla like radial scanning.

From the inferior duodenal angle

Using stretch technique, the convex scope reaches to the inferior duodenal angle, we should suck air from the duodenal lumen, and fill $50-100 \mathrm{ml}$ of deareated water, if necessary. At this point, there are two big transeverse ductal structure in the lower half, which are the aorta and vena cava (Fig. 18). Then we pull the scope gradually after doing up-angle to the lesser curvature of

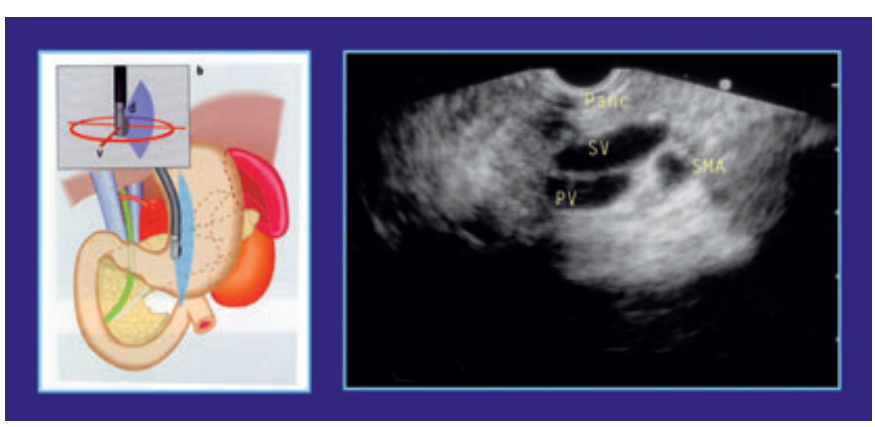

Fig. 13 From the stomach.

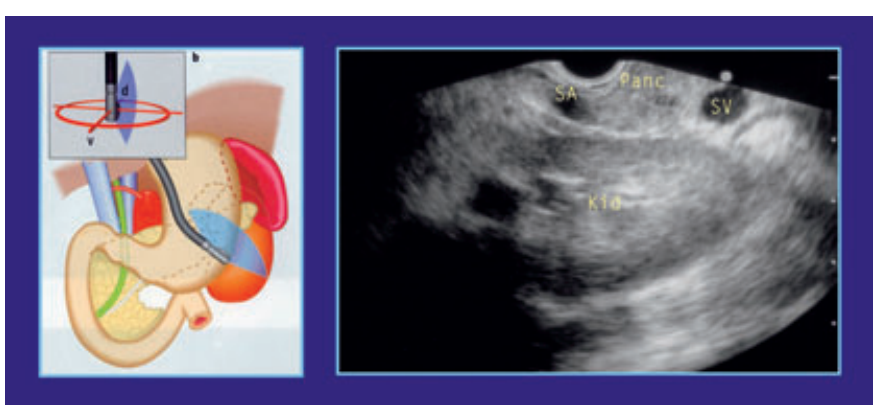

Fig. 14 From the stomach.

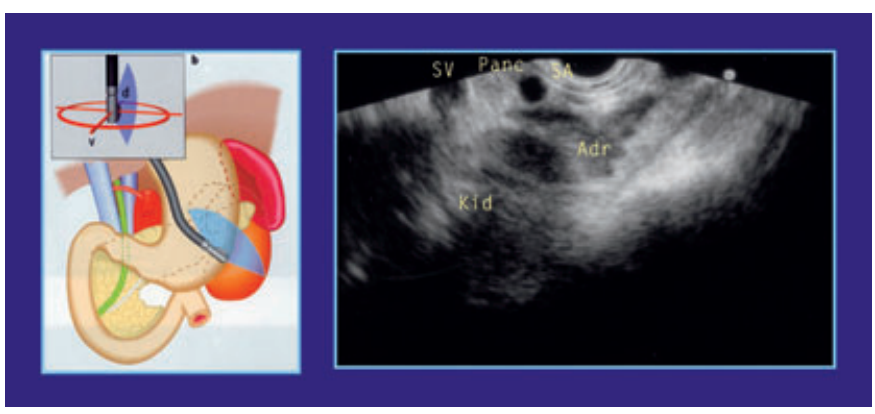

Fig. 15 From the stomach. 


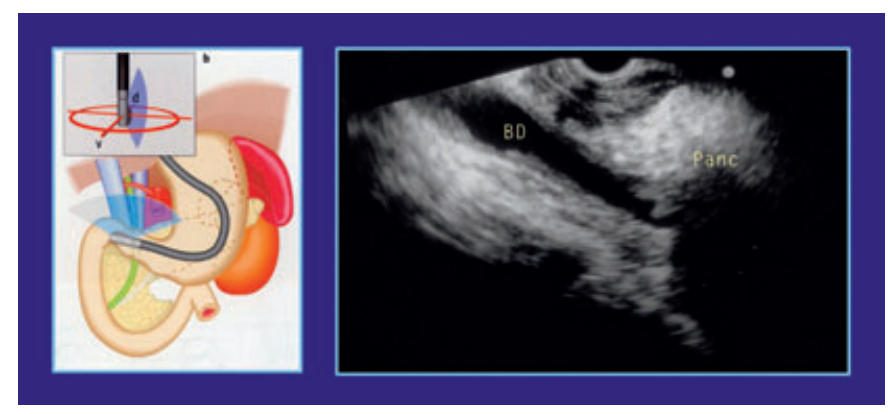

Fig. 16 From the duodenal bulb.

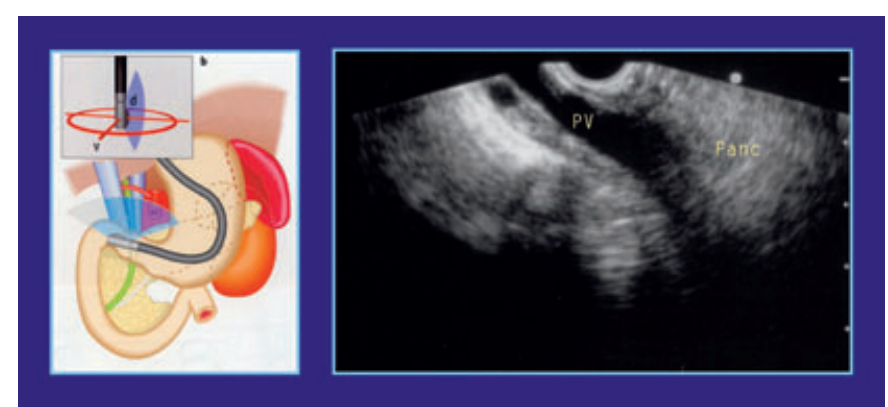

Fig. 17 From the duodenal bulb.

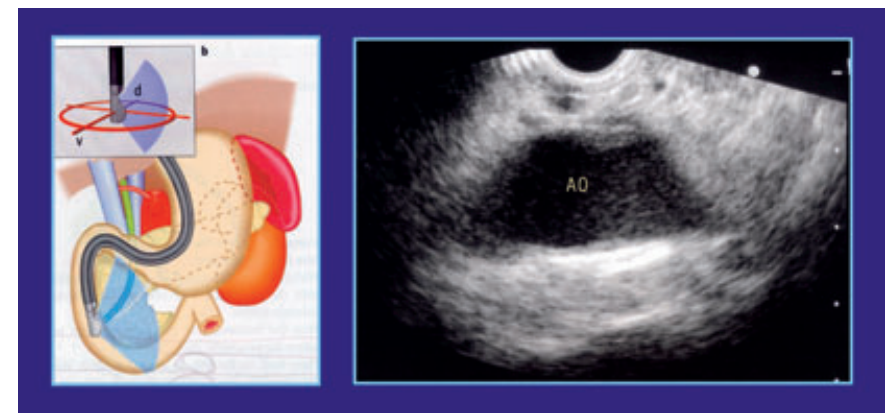

Fig. 18 From the inferior duodenal angle.

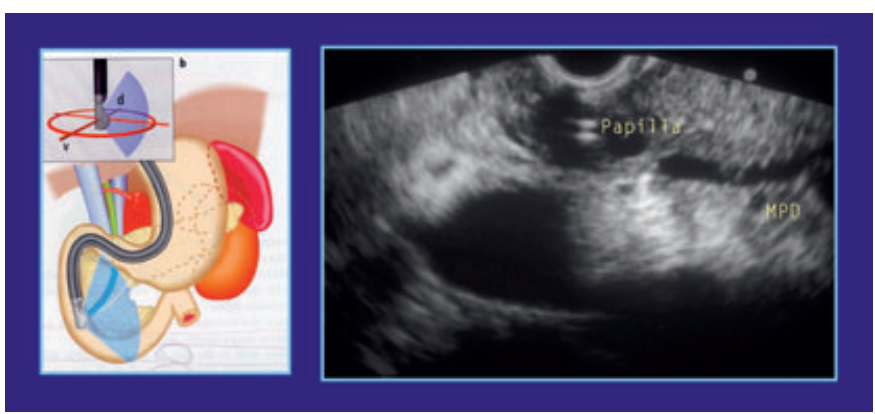

Fig. 19 From the inferior duodenal angle.

the duodenum and will find thickening of the duodenal wall (papilla) and two ductal structure (bile duct and main pancreatic duct) that are startng from the papilla (Fig. 19). However using this pulling method, it is difficult to visualize the transitional part of the pancreas head-body, because the scope is easy to slip out.

\section{Conclusions}

EUS has great potential, diagnostic and treatment capability, however it is most important to know the anatomy of biliopancreatic system.

\section{References}

${ }^{1}$ Kida M. Endoscopic ultrasonography in Japan: Present status and standardization. Digest Endosc 2002; 14 (suppl): 24-29

2 Inui K, Kida M, Fujita N, Maguchi H, Yasuda K, Yamao K. Standard Radial Imaging Techniques in the Pancreatobiliary Region Using Endoscopic Ultrasonography 